\title{
Extreme value statistics in Raman fiber lasers
}

\author{
Dmitriy V. Churkin $^{* a, b}$, Oleg A. Gorbunov ${ }^{a}$, Sergey V. Smirnov ${ }^{b}$ \\ a Institute of Automation and Electrometry, SB RAS, Novosibirsk 630090, Russia; \\ ${ }^{\mathrm{b}}$ Novosibirsk State University, Novosibirsk, 630090, Russia
}

\begin{abstract}
We present the numerical study of the statistical properties of the partially coherent quasi-CW high-Q cavity Raman fiber laser. The statistical properties are different for the radiation generated at the spectrum center or spectral wings. It is found that rare extreme events are generated at the far spectral wings at one pass only. The mechanism of the extreme events generation is a turbulent-like four-wave mixing of numerous longitudinal generation modes. The similar mechanism of extreme waves appearance during the laser generation could be important in other types of fiber lasers.
\end{abstract}

Keywords: fiber lasers; extreme statistics; nonlinear optics; photon statistics.

\section{INTRODUCTION}

Partially coherent (PC) quasi-CW fiber lasers constitutes a wide range of laser sources including Ytterbiumdoped fiber lasers (YDFLs), Bi-doped fiber lasers, Tm-doped fiber lasers, lasers based on other types of active fibers and Raman fiber lasers (RFLs). RFLs are well-known robust fiber laser sources providing almost any wavelength in the near-IR range (1.1-1.7 mkm) [1]. In addition, RFLs can easily generate multiple wavelengths simultaneously [2], their output radiation can be tuned over a wide frequency range in an all-fiber configuration by using tunable FBGs [3,4] or by using tunable pump source [5]. All these features make RFLs useful in various fields including supercontinuum generation [6] and medical applications [7-8]. Recently, the visible radiation has been generated by frequency doubling of the RFL $[9,10]$.

A typical feature of RFLs is a long cavity having the length starting from several meters to hundreds of kilometers [11]. Typical spectral width of output radiation is about 0.1-1 nm. Thus, the output radiation of conventional RFL consists of numerous longitudinal modes, up to $10^{8}$ [12]. Longitudinal modes interact with each other in the long nonlinear and dispersive RFL cavity [13]. As a result, the time dynamics of the RFL is complex with non-trivial statistical properties [14] probably revealing existence of rare intense events having probability higher than prescribed by Gaussian statistics.

In general, extreme type statistics in nonlinear fiber optics has attracted a great attention since the experimental demonstration of optical rogue waves in supercontinuum (SC) generation [15]. Up to date, the extreme events statistics is observed in many optical systems including pulsed and cw pumped SC sources [16], silicon [17] and fiber [18] Raman amplifiers, optical filamentation [19] and others.

In different systems there are different physical mechanisms attributed to the emergence of the rare intense events. The extreme events are defined by the interplay of the modulation instability, third-order dispersion and collisions of Raman-shifted solitons in SC sources [16, 20]. The formalism of Akhmediev breathers provides the sufficient advance in understanding of physics of rogue waves in optical system [21-23] including classical problems of rogue waves appearance on a water surface [24]. In Raman fiber amplifiers (RFAs) [18] and Raman parametric amplifiers [25], the main mechanism is the exponential transfer of the intensity fluctuations from the pump wave to the signal via Raman response which can be significantly enhanced in the case of the weak walk-off parameter [18]. Similar, in the optical filamentation processes the noise transfer from the pump plays a key role [19]. Also it has been shown, that sporadic rogue waves events emerging from turbulent fluctuations as bursts of light during propagation of the optical wave along the thousands of nonlinear lengths in anoumalous dispersion fiber could exist [26].

In the present paper, we study the emergence of intense rare events in the generation of the $\mathrm{PC}$ quasi-CW fiber laser on the example of the high-Q cavity RFL operating in the normal dispersion regime.

* churkin@iae.nsk.su; phone 738333282 54; fax 73833328254.

Fiber Lasers IX: Technology, Systems, and Applications, edited by Eric C. Honea,

Proc. of SPIE Vol. 8237, 82372W · ( 2012 SPIE · CCC code: 0277-786X/12/\$18 · doi: 10.1117/12.907363

Proc. of SPIE Vol. 823782372 W-1 


\section{NUMERICAL MODEL}

To simulate laser generation we use a model based on the NLSE:

$$
\begin{aligned}
& \frac{\partial A_{p}^{ \pm}}{\partial z}+\frac{i}{2} \beta_{2 p} \frac{\partial^{2} A_{p}^{ \pm}}{\partial t^{2}}+\frac{\alpha_{p}}{2} A_{p}^{ \pm}=i \gamma_{p}\left(\left|A_{p}^{ \pm}\right|^{2}+\left(2-f_{R}\right)\left|A_{s}^{ \pm}\right|^{2}\right) A_{p}^{ \pm}-\frac{g_{p}}{2}\left(\left|A_{s}^{ \pm}\right|^{2}+\left\langle\left|A_{s}^{\mp}\right|^{2}\right\rangle\right) A_{p}^{ \pm} \\
& \frac{\partial A_{s}^{ \pm}}{\partial z}+\left(\frac{1}{v_{s}}-\frac{1}{v_{p}}\right) \frac{\partial A_{s}^{ \pm}}{\partial t}+\frac{i}{2} \beta_{2 s} \frac{\partial^{2} A_{s}^{ \pm}}{\partial t^{2}}+\frac{\alpha_{s}}{2} A_{s}^{ \pm}=i \gamma_{s}\left(\left|A_{s}^{ \pm}\right|^{2}+\left(2-f_{R}\right)\left|A_{p}^{ \pm}\right|^{2}\right) A_{s}^{ \pm}+\frac{g_{s}}{2}\left(\left|A_{p}^{ \pm}\right|^{2}+\left\langle\left|A_{p}^{\mp}\right|^{2}\right\rangle\right) A_{s}^{ \pm}
\end{aligned}
$$

where $\mathrm{A}$ is a complex field envelope, $\mathrm{z}$ is a coordinate, $\mathrm{t}$ stands for time in a frame of references moving with pump wave, vs vp are pump and Stokes waves group-velocities, $\beta_{2}, \alpha, \gamma, g$ are dispersion, linear attenuation, Kerr and Raman coefficients, \pm denotes counter-propagating waves, "s" and "p" are used for Stokes and pump waves. The complementary approach is to re-write the NLSEs set in the terms of the longitudinal modes [27].

Several general assumptions are used in the model. First of all, we do not include in our model such processes as SBS and Rayleigh scattering as they do not contribute sufficiently to the generation of conventional RFL having the cavity based on the fiber Bragg gratings (FBGs). However, the Rayleigh scattering should be taken into account if one would like to describe the generation properties of recently developed random distributed feedback fiber lasers [28] operating via Raman-amplified distributed Rayleigh backscattering and having no FBGs. We also assume that Raman gain is instantaneous, thus the model can not describe any time dynamics faster than $1 \mathrm{ps}$ as the typical Raman response time is about $100 \mathrm{fs}$. Raman gain is wavelength independent in our model as typical spectrum width $(1 \mathrm{~nm})$ is much less than typical Raman gain scale $(10 \mathrm{~nm})$. We have checked that taking into account the Raman gain dependence on wavelength does not affect main results obtained in this paper.

In the laser cavity, two counter-propagating waves are simultaneously and self-consistently generated, and one needs to know intensities of both waves to calculate their values at the next numerical step. Totally, there are 4 coupled NLSEs for pump and Stokes waves. We can simplify machinery by averaging the intensities of counterpropagating waves since these waves move fast with regard to each other thus do not contributing to the spectral broadening inducing time independent phase incursion only. After that, we integrated Eqs. along z using iterative approach, i.e. when integrating equations for $\mathrm{A}+\mathrm{s}, \mathrm{p}$ we used $\mathrm{A}-\mathrm{s}, \mathrm{p}$ obtained on previous iteration, and vice versa. Similar approach is used in [29-31].

The following fiber parameters were used in simulations: $\alpha_{\mathrm{p}}=0.5(\mathrm{~km})^{-1}, \alpha_{\mathrm{s}}=0.83(\mathrm{~km})^{-1}, \beta_{2 p}=17.9 \mathrm{ps}^{2} / \mathrm{km}$, $\beta_{2 \mathrm{~s}}=7.17 \mathrm{ps}^{2} / \mathrm{km}, \gamma_{\mathrm{s}}=3(\mathrm{~km} \mathrm{~W})^{-1}, \mathrm{~g}_{\mathrm{s}}=1.3(\mathrm{~km} \mathrm{~W})^{-1}$, laser cavity length $\mathrm{L}=370 \mathrm{~m}$, pump power $3 \mathrm{~W}$. Laser mirrors were modeled by super-gaussian FBGs of $0.5 \mathrm{~nm}$ width. We used white noise as initial conditions for Stokes wave ( 1 photon with random phase in each mode). Results of modeling do not depend on grid size at least in the range of $2^{12-2^{20}}$ grid points. We have checked that nor decreasing the time steps, nor increasing the window affect the results.

Effectively, we model the laser generation by using the propagation equations instead of solving the boundary value problem. From this point of view, our system seems to be like a Raman amplifier. However, it is really different because we deal with the laser generation, but not a signal amplification that results in a different physical mechanisms responsible for the properties of the generated radiation. In particular, in our case of the laser generation the exponential transfer of the fluctuations from the pump wave to the generation wave (XPM between pump and Stokes waves) has no influence on the temporal and statistical properties of the generated radiation well above the generation threshold what has been carefully checked. The corresponding term proportional to $\gamma A_{s} A_{p}^{2}$ could be omitted in equations.

\section{SPECTRAL AND STATISTICAL PROPERTIES}

The generation spectrum well above the threshold is wider than the spectral profiles of the FBGs, Fig. 1. Thus, there are two principally different parts in the spectrum: The central part and the spectral wings. As the FBGs are highly reflective, the central part of the spectrum propagates over many cavity passes. Turbulent-like four-wave mixing processes [13] lead to the generation of far spectral wings. Being detuned from the spectrum centre more than FBGs width, the far spectral wings leave the cavity at each laser mirror and, therefore, are generated at one 
fiber pass only. The statistical properties of the intra-cavity radiation in general should be defined mainly by the central part rather than by the spectral wings as the power in the central part is several times higher than the power in the wings. On the contrary, the statistical properties of the output radiation should be defined mainly by the spectral wings as the central part is filtered out by the FBG.

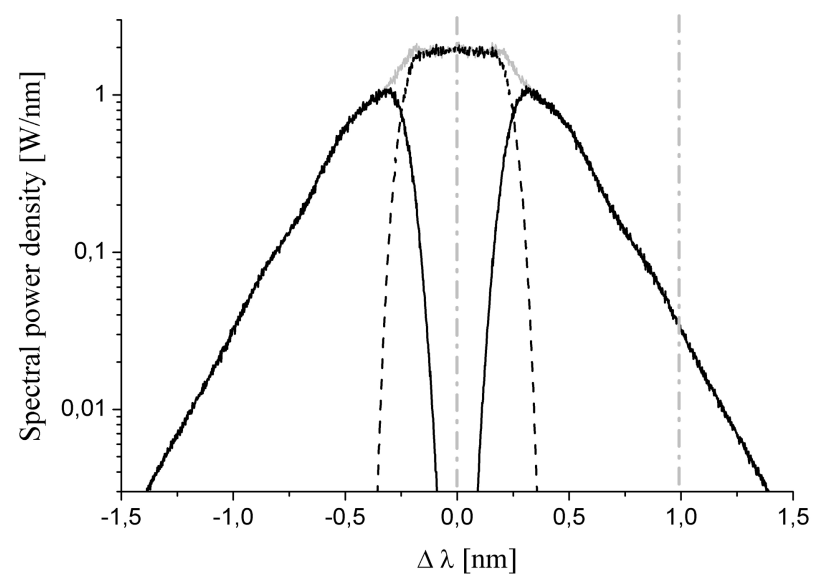

Fig.1. Total intra-cavity (gray line), reflected from the FBG (dashed line) and output (black line) spectrum generated in high-Q cavity RFL. Vertical mixed lines indicate the central positions of two rectangular spectral filters.
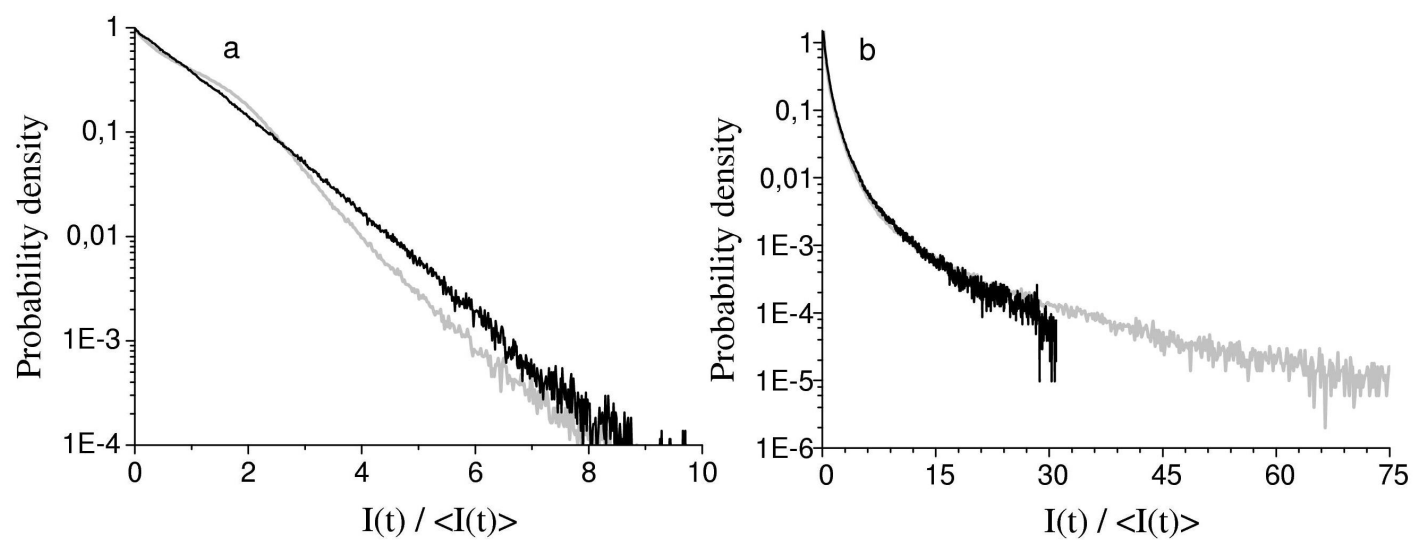

Fig. 2 Intensity PDF (a) and ACF (b) of the total intra-cavity (gray line), reflected from the FBG (dashed line) and output (black line) radiation.

We found that the statistical properties are sufficiently different for the intra-cavity and output spectrum, Fig.2a, i.e. the statistical properties of the radiation generated at one pass and many cavity passes are different. The intensity auto-correlation function (ACF) confirms the different nature of intra-cavity and output radiation, Fig. 2b. Note that the difference in statistical properties of incident and reflected back from the FBG radiation has been pointed in [29].

The intensity PDF of the intra-cavity radiation is sufficiently non-exponentional revealing that correlations between different longitudinal modes do exist [14]. Using the rectangular spectral filters of different spectral widths and spectral positions (the filter 1 is centered at the spectrum center, the filter 2 is detuned by $1 \mathrm{~nm}$ from the spectrum center), we calculate the intensity PDF of specific part of the generation spectrum. It is found that the intensity PDF of the modes generated on the flat top at the spectrum center is exponential, Fig. 3a Increasing the filter width, the PDF becomes more and more non-exponential, compare Fig. 3a with gray curve Fig. 2a (corresponds to full spectral window, i.e. $\sim 5 \mathrm{~nm}$ filter). Therefore, neighboring longitudinal modes at the central part of the spectrum are generated independently, i.e. they are uncorrelated. Contrary to the intuitive consideration that in the dispersive media closely spaced spectral components are more likely to be correlated than far away spaced components, exactly distant spectral components have to be correlated in the RFL. A physical reason of such far correlations is unclear. 

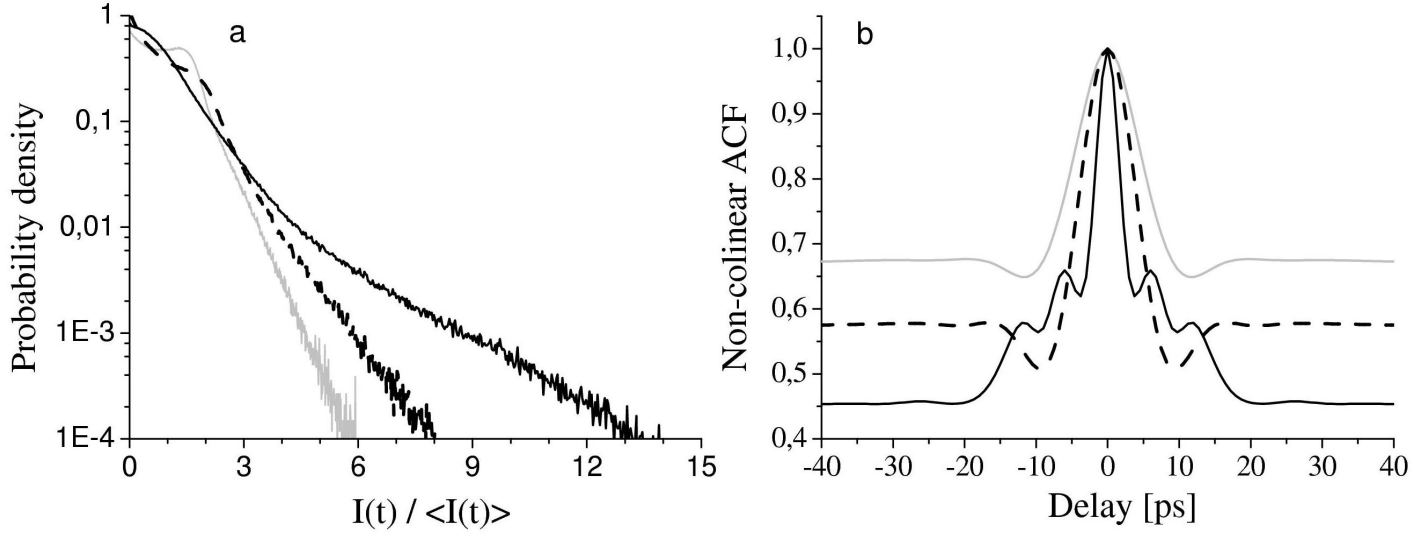

Fig. 3 Intensity PDF of the modes filtered out from the (a) spectrum center and (b) spectral wing (filter is detuned by $1 \mathrm{~nm}$ from the spectrum center). Filters spectral full widths are $0.1 \mathrm{~nm}$ (black) and $0.5 \mathrm{~nm}$ (gray).

Intensity $\mathrm{PDF}$ of spectral components at far spectral wings are sufficiently non-exponential revealing the existence of intense rare events having the probability higher than probability defined by the Gaussian distribution, Fig. 3b. The extreme rare events are more pronounced in the output radiation of the high-Q cavity RFL. The self-filtering of extreme events by the laser mirror is similar to the spectral filtering approach used previously in Raman fiber amplifiers [32].

The temporal behavior of the total intra-cavity radiation is irregular with some rare events having the amplitude several times higher than the mean value, Fig.4a. The intensity evolution of the radiation within the narrow spectral region at the spectrum center is similar, Fig. 4b. Such intensity evolution is typical for stochastic signal which consists of many independently generated modes obeying the Gaussian distribution.

The output radiation comprises the events having the amplitude $>100$ times higher than the average output power in the given spectral region, Fig.4c. Note, that extreme events could be seen in the total radiation (compare, for example, pikes near 3700 ps at Fig. 4a and Fig. 4c), having amplitude $>6$ times higher than total average power. The typical temporal width of the extreme rare event at the laser output is $\sim 10 \mathrm{ps,} \mathrm{see} \mathrm{ACF} \mathrm{at} \mathrm{Fig.} 2 \mathrm{~b}$, that makes difficult their direct detection in real-time as these events are generated irregularly.

The extreme rare events are generated in RFL at far spectral wings thus being generated in one fiber pass only. However, the physical mechanism of rare events emergences in RFL is different from those observed in RFA. In an amplifier, the signal propagating along the fiber acquires the intensity fluctuations from the pump wave which can be evolved into the extreme pulses under conditions of self-induced modulation instability [18]. Contrary, the noise transfer from the pump wave to the generation wave has no influence on statistical properties of RFL. The rare events are generated in turbulent four-wave mixing interaction of numerous longitudinal modes and located at far spectral wings that could be potentially described using the formalism of a weak wave turbulence that should be further investigated.

In the RFL the pump wave is sufficiently depleted that is taken into account by the simultaneous modeling of NLSEs for the generated and the pump wave. However, the strong depletion of the pump wave does not imply the
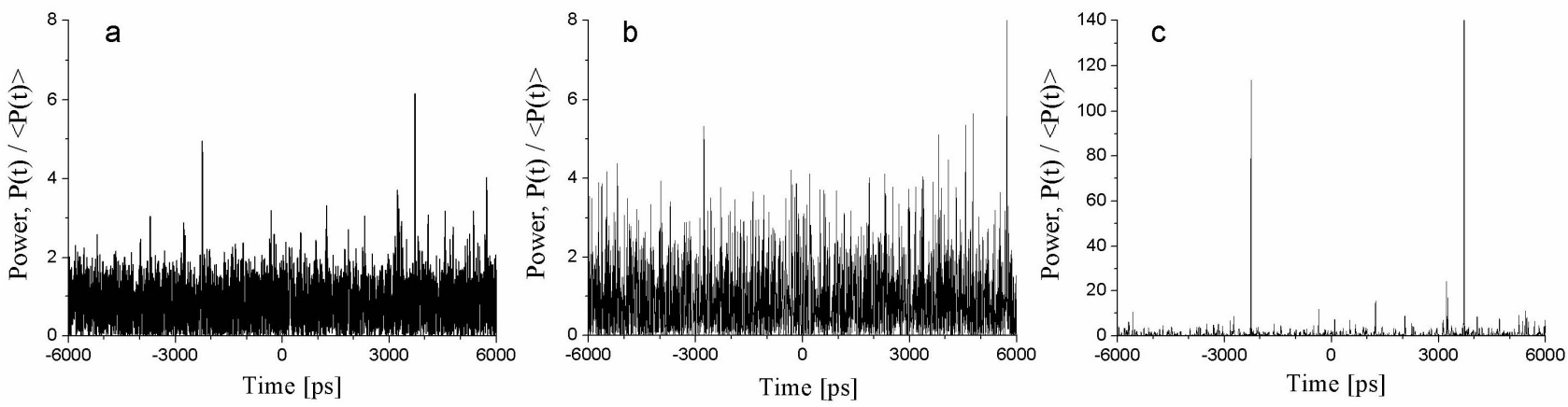

Fig. 4 (a) The time dynamics of the total intra-cavity radiation as well as radiation generated within the $0.5 \mathrm{~nm}$ spectral width at the (b) spectrum center and (c) far spectral wing. 
restrictions on the PDF: the intensity PDF tails in RFL does not asymptotically approach the pump wave exponential PDF as opposite to the case of RFA where the pump depletion plays a critical role [33].

\section{CONCLUSION}

To conclude, using the NLSE-based numerical modeling, we have found that the radiation of partially coherent quasi-CW RFL consist of two different parts which have different statistical properties. The central spectral part of the radiation comprises longitudinal modes being uncorrelated on small wavelength detunings. A physical mechanism of far spectrally detuned mode correlations should be further clarified. Far spectral wings are generated at one fiber pass only. The intensity PDFs of spectral wings reveal the existence of extreme rare events. As the extreme rare events are generated at far spectral wings, they are more pronounced at the laser output being self-filtered out by laser mirrors. The main physical mechanism of extreme event emergence in Raman fiber laser is turbulent-like four-wave mixing processes between numerous longitudinal modes in generation. The similar mechanism of extreme waves appearance during the laser generation could be important in other types of fiber lasers including random distributed feedback fiber lasers operating via Raman [28] or Brillouin [34] gain.

The authors acknowledge support from the Russian Ministry of Science and Education, a research grant of the Russian Federation Government N11.G34.31.0035, non-governmental Dynasty foundation, and thank E.V. Podivilov for fruitful discussions. The computations were performed at the Novosibirsk State University Scientific computing centre.

\section{REFERENCES}

[1] E.M. Dianov, I.A. Bufetov, M.M. Bubnov, M.V. Grekov, S.A. Vasiliev, and O.I. Medvedkov, "Three-cascaded 1407-nm Raman laser based on phosphorus-doped silica fiber," Opt. Lett. 25, 402 (2000) .

[2] Y.-G. Han, C.-S. Kim, J.U. Kang, U.-C. Paek, Y. Chung, "Multiwavelength Raman Fiber Ring Laser Based on Tunable Cascaded Long-Period Fiber Gratings," IEEE Photon. Technol. Lett., 15, 383 (2003).

[3] P. C. Reeves-Hall and J. R. Taylor, "Wavelength and duration tunable sub-picosecond source using adiabatic Raman compression," Electron. Lett. 37, 417 (2001).

[4] S.A. Babin, D.V. Churkin, S.I. Kablukov, M.A. Rybakov, and A.A. Vlasov, "All-fiber widely tunable Raman fiber laser with controlled output spectrum," Opt. Express 15, 8438 (2007).

[5] V. A. Akulov, D. M. Afanasiev, S. A. Babin, D. V. Churkin, S. I. Kablukov, M. A. Rybakov, A. A. Vlasov, Laser Physics, "Frequency tuning and doubling in Yb-doped fiber lasers," Laser Phys. 17(2), 124 (2007).

[6] A.K. Abeeluck, C. Headley, and C.G. Jorgensen, "High-power supercontinuum generation in highly nonlinear, dispersion-shifted fibers by use of a continuous-wave Raman fiber laser," Opt. Lett. 29, 2163 (2004).

[7] A. S. Kurkov, V. M. Paramonov, O.I. Medvedkov, I. D. Zalevskii, S. E. Goncharov, "Fiber Raman laser at $1450 \mathrm{~nm}$ for medical applications," Laser Physics, 18,1234 (2008)

[8] A. S. Yusupov, S. E. Goncharov, , I. D. Zalevskii, V. M. Paramonov, A. S. Kurkov, Laser Physics, "Raman fiber laser for the drug-free photodynamic therapy," Laser Phys. 20, 357 (2010).

[9] D. Georgiev, V. P. Gapontsev, A. G. Dronov, M. Y. Vyatkin, A. B. Rulkov, S. V. Popov, and J. R. Taylor, "Watts-level frequency doubling of a narrow line linearly polarized Raman fiber laser to 589nm", Opt. Express 13, $6772(2005)$.

[10] S. I. Kablukov, S. A. Babin, D. V. Churkin, A. V. Denisov and D. S. Kharenko, "Frequency doubling of a Raman fiber laser" Laser Phys. 20, 365 (2010).

[11] S. K. Turitsyn, J. Ania-Castañón, S.A. Babin, V. Karalekas, P. Harper, D. V. Churkin, S. I. Kablukov, A. E. ElTaher, E.V. Podivilov, and V. K. Mezentsev, "270-km Ultralong Raman Fiber Laser", Phys. Rev. Lett. 103, 133901 (2009).

[12] S.A. Babin, V. Karalekas, E.V. Podivilov, V.K. Mezentsev, P. Harper, J. Ania-Castañón, and S.K. Turitsyn, "Turbulent broadening of optical spectra in ultralong Raman fiber lasers" Physical Review A 77, 033803 (2008).

[13] S. A. Babin, D. V. Churkin, A. E. Ismagulov, S. I. Kablukov, E. V. Podivilov, "Four-wave-mixing-induced turbulent spectral broadening in a long Raman fiber laser", J. Opt. Soc. Am. B, 24, 1729 (2007) 
[14]D.V. Churkin, S.V. Smirnov, and E.V. Podivilov, "Statistical properties of partially coherent cw fiber lasers.," Optics letters, 35, 3288 (2010).

[15] D.R. Solli, C. Ropers, P. Koonath, B. Jalali, “Optical Rogue Waves”, Nature 450, 1054 (2007).

[16] J.M. Dudley, G. Genty, and B.J. Eggleton, "Harnessing and control of optical rogue waves in supercontinuum generation," Optics Express, 16, 3644 (2008).

[17]D. Bourlaug, S. Fathpour, B. Jalali, "Extreme Value Statistics in Silicon Photonics", IEEE Photonics J. 1, 33 (2009)

[18] K. Hammani, C. Finot, J.M. Dudley, G. Millot, "Optical rogue-wave-like extreme value fluctuations in fiber Raman amplifiers" Opt. Express 16, 16467, (2008)

[19] J. Kasparian, P. Bejot, J.P. Wolf, J.M. Dudley, "Optical rogue wave statistics in laser filamentation" Opt. Express 17(14), 12070 (2009)

[20] A. Mussot, A. Kudlinski, M. Kolobov, E. Louvergneaux, M. Douay, and M. Taki, "Observation of extreme temporal events in CW-pumped supercontinuum.," Opt. Express, 17, 17010 (2009).

[21]N. Akhmediev, A. Ankiewicz, and J. Soto-Crespo, "Rogue waves and rational solutions of the nonlinear Schrödinger equation,” Physical Review E, 80, 026601 (2009).

[22] N. Akhmediev, J.M. Soto-Crespo, and A. Ankiewicz, "Extreme waves that appear from nowhere: On the nature of rogue waves," Physics Letters A, 373, 2137 (2009).

[23] J.M. Dudley, G. Genty, F. Dias, B. Kibler, and N. Akhmediev, "Modulation instability, Akhmediev Breathers and continuous wave supercontinuum generation.," Optics express, 17, 21497 (2009).

[24] Chabchoub, N. Hoffmann, and N. Akhmediev, "Rogue Wave Observation in a Water Wave Tank," Physical Review Letters, 106, 204502 (2011).

[25] K. Hammani, C. Finot, G. Millot, "Emergence of extreme events in fiber-based parametric processes driven by a partially incoherent pump wave" Opt. Lett. 34, 1138 (2009)

[26] K. Hammani, B. Kibler, C. Finot, and A. Picozzi, "Emergence of rogue waves from optical turbulence," Physics Letters A, 374, 3585 (2010)

[27]E. G. Turitsyna, G. Falkovich, V. K. Mezentsev, and S. K. Turitsyn, "Numerical investigation of the impact of reflectors on spectral performance of Raman fibre laser," Physical Review A, 80, 031804 (2009).

[28] S.K. Turitsyn, S.A. Babin, A.E. El-Taher, P. Harper, D.V. Churkin, V. Karalekas, E.V. Podivilov, S.I. Kablukov, and J.D. Ania-Castanon, "Random distributed feedback fibre laser", Nature Photonics 4, 231 (2010).

[29] S. Randoux, N. Dalloz, and P. Suret, "Intracavity changes in the field statistics of Raman fiber lasers.," Optics letters, 36, 790 (2011).

[30] S.K. Turitsyn, A.E. Bednyakova, M.P. Fedoruk, A.I. Latkin, A.A. Fotiadi, A.S. Kurkov, and E. Sholokhov, "Modeling of CW Yb-doped fiber lasers with highly nonlinear cavity dynamics," Optics Express, 19, 1227 (2011).

[31]C. E. Preda, G. Ravet, A. A. Fotiadi, and P. Mégret, "Iterative method for Brillouin fiber ring resonator," inCLEO/Europe and EQEC 2011 Conference Digest, OSA Technical Digest (CD) (Optical Society of America, 2011), paper CJ_P27.

[32] C. Finot, K. Hammani, J. Fatome, J.M. Dudley, and G. Millot, "Selection of Extreme Events Generated in Raman Fiber Amplifiers Through Spectral Offset Filtering,” IEEE Journal of Quantum Electronics, 46, 205-213 (2011)

[33] K. Hammani, A. Picozzi, and C. Finot, "Extreme statistics in Raman fiber amplifiers: From analytical description to experiments" Opt. Comm. 284, 2594 (2011).

[34] A. Fotiadi, E. Preda, and P. Mégret, "Brillouin Fiber Laser with Incoherent Feedback," in CLEO: 2011 - Laser Applications to Photonic Applications, OSA Technical Digest (CD) (Optical Society of America, 2011), paper CTul6. 